\title{
Appareil
}

$10 \mid 2012$

Lyotard et la surface d'inscription numérique

\section{Lyotard, penseur du différend culturel}

\author{
Jean-Louis Déotte
}

\section{OpenEdition}

Journals

Édition électronique

URL : http://journals.openedition.org/appareil/115

DOI : 10.4000/appareil. 115

ISSN : 2101-0714

Éditeur

MSH Paris Nord

Référence électronique

Jean-Louis Déotte, «Lyotard, penseur du différend culturel », Appareil [En ligne], 10 | 2012, mis en ligne le 09 février 2008, consulté le 30 juillet 2020. URL : http://journals.openedition.org/appareil/115 ; DOI : https://doi.org/10.4000/appareil.115

Ce document a été généré automatiquement le 30 juillet 2020.

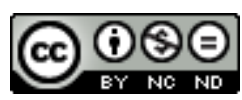

Appareil est mis à disposition selon les termes de la Licence Creative Commons Attribution - Pas d'Utilisation Commerciale - Pas de Modification 4.0 International. 


\title{
Lyotard, penseur du différend culturel
}

\author{
Jean-Louis Déotte
}

«Dans un monde où nous sommes condamnés à
une fluidité généralisée, le métissage mondial des
cultures, la world music, etc., semblent aller de
soi. Ce qui nous désarme devant des attitudes de
vie, des évaluations cultuelles et culturelles
incompréhensibles. Il est urgent de replacer le
concept de différend au centre de la vie
publique "

1 Quand Lyotard aborde la question du différend ${ }^{1}$, ce n'est pas pour enrichir une quelconque science de l'interculturel. Lyotard part d'une situation critique dans tous les sens du terme critique (krinein) : la fin des années 1970 est caractérisée en France par un antisémitisme insidieux. Papon avait été ministre de Giscard, dont le premier Ministre R. Barre avait eu cette réaction pour le moins étonnante après l'attentat contre la synagogue de la rue Copernic, faisant la distinction, revendiquée jusqu'à sa mort, entre les victimes « innocentes » (non juives) et les autres... Période marquée par une montée en puissance du négationnisme qui infecte tous les milieux intellectuels et en particulier une partie de l'extrême gauche. Il était très tendance d'être négationniste. La situation était en plus critique parce qu'il y allait du jugement et de la faculté de juger, question qui sera au cœur de la Décade de Cerisy organisée autour du travail de Lyotard en 1982. Elle était critique parce que les négationnistes faisaient à leur manière de l'histoire, certes comme des historiens pervers, car ils ne cessaient de réclamer la preuve de la preuve, mais n'était-ce point pousser l'historiographie dans ses retranchements? Il n'y avait pas plus grands défricheurs d'archives qu'eux, pas de plus grands positivistes. En fait la situation était critique, parce qu'ils avaient réussi à introduire un virus dans la machine historiographique : sa propre méthodologie.

2 La démarche de Lyotard va consister à démonter le cercle dans lequel ils voulaient nous enfermer. Lyotard va montrer ainsi que les négationnistes poursuivent le crime de 
disparition de masse réalisé par les nazis, mais surtout il va montrer l'impuissance de la raison historienne : il y a des victimes de l'histoire qu'aucun tribunal ne peut entendre, parce que le tort subi ne peut pas devenir un litige accepté comme tel par un tribunal de la raison. En cela Lyotard poursuit l'inspiration du dernier Benjamin, et en particulier sa fameuse critique du " concept d'histoire ${ }^{2}$ ». Le Différend s'inscrit dans la polémique menée alors par Lyotard contre l'éthique communicationnelle d'Habermas. Pour les habermassiens qui faisaient alors la loi dans l'Université allemande, il y a un critère absolu pour écarter tel ou tel locuteur d'une communauté langagière : est-il d'accord ou non pour accepter les conditions langagières d'établissement de la vérité ? Ces conditions reprennent en les élargissant un certain nombre de réquisits énoncés par Kant dans la Faculté de juger au titre du sensus communis.

3 Kant définit le «sens commun »: «1) Penser par soi-même ; 2) Penser en se mettant à la place de tout autre ;3) Toujours penser en accord avec soi-même ${ }^{3}$ ».

4 Dans une acception classique du sensus communis on a les exigences de ce que le tribunal de la raison pose pour n'importe quel témoin ou n'importe quel prévenu : pouvez-vous donner les raisons de votre acte? On retrouve ici le principe de raison élaboré par Leibniz. Soit : êtes-vous autonome (autonome au sens du respect de la loi universelle)? Pouvez-vous sur le plan de l'éthique, universaliser les mobiles de votre action? Êtesvous sincère avec vous-même (aspect religieux de la conscience réformée)?

Qui dès lors sera exclu du cercle de la communication véridique, scientifique, démocratique, "authentique"? Celui ou celle qui se place dans une position d'hétéronomie parce qu'il accorde foi à une parole révélée (le juif, le chrétien, le musulman, etc.), qui donc se trouve obligé par elle, celui ou celle qui agit traditionnellement, comme le font ceux qui de tout temps sont sous le magistère des grands ancêtres mythiques communautaires, parce qu'on a toujours agi comme cela icibas. Ces locuteurs transmettant les histoires qu'ils ont toujours entendu raconter et en appliquent, sous la forme d'un rituel, les codes.

6 Lyotard est le grand penseur de notre situation post-coloniale, parce qu'il isole des normes, c'est-à-dire des pôles de légitimité, c'est-à-dire des rapports à la loi, qui sont indérivables les uns des autres. On ne peut pas déduire la légitimité de l'une de celle d'une autre. Il y a trois pôles de légitimité : le narratif, la révélation ou obligation, la délibération, ce qui permet de distinguer trois grands modes de l'être-ensemble: les sociétés sauvages et païennes, les sociétés du théologico-politique, la société démocratique-capitaliste, trois rapports au corps : l'inscription de la loi sur le corps, l'incarnation, l'objectivation, trois surface d'inscription: la marque et ses supports, l'incorporation, la représentation. Dès lors on dira qu'il y a différend quand un locuteur respectant l'une de ces normes se trouvera comme chez Kafka dans l'incapacité de se faire entendre par le tribunal de l'autre norme. Or la mondialisation a quasiment universalisé la norme de délibération au détriment des autres normes. L'exportation de la démocratie la plus superficielle (des élections, un parlement élu et le tour est joué) va dans ce sens. C'est toute la question du développement qui est en jeu : qu'est-ce qu'un système d'élections sans culture démocratique? Sans participation effective de tous à la vie politique?

D'où la multiplication des situations de différend, même en Occident. J'en veux pour preuve la quotidienneté des affaires jugées par nos tribunaux correctionnels, voire en Assises, où le prévenu, par exemple issu de l'immigration, ne peut rendre raison de ses actes parce qu'il respecte sa propre norme. D'un point de vue immédiat, c'est une 
mésentente comme le dit Rancière : prévenu et tribunal utilisent les mêmes mots, en apparence partagent le même idiome, mais en donnant aux mots un sens différent. Bel exemple : le film de Rozier: Maine océan. Dans une affaire de coups et blessures, le pêcheur breton qui patoise désarçonne le tribunal condamné de ce fait à rire et à le relaxer. En fait, il y a là en plus un vrai différend: entre l'homme de loi muni de son code et le païen adorant raconter des histoires.

Il y a un glissement argumentatif dans Le Différend: le point d'attaque de la démonstration de Lyotard, c'est l'évidente incapacité des victimes de la Shoah de se faire entendre. On ne témoigne pas d'avoir été gazé, on a bien du mal à raconter ce qu'on a subi dans l'humiliation. Les raisons de se taire sont multiples, je ne les rappelle pas ici. On pourrait penser à partir de là que Lyotard vient conforter la réorientation de l'audience publique sur la victime comme le prône notre président de la République. En fait, il n'y a pas que des victimes dans la série lyotardienne, il y a un Antillais et une masse d'Algériens ${ }^{4}$ qui ne voient pas pourquoi ils devraient respecter les devoirs des Français, il y a un prolétaire que le tribunal des Prud'hommes déboute parce qu'il ne trouve pas « naturel » de louer sa force de travail comme tout salarié qui se respecte, il y a des fantassins italiens opposés aux Autrichiens lors des batailles alpines de la sanglante première guerre mondiale et qui enchaînent sur l'ordre lancé d'attaquer en applaudissant leur héroïque officier, tout en restant blotti au cœur de leur tranchée... Il y a bien d'autres personnages comme cela, que Lyotard reconnaissait comme subissant un tort : une malienne exciseuse de sa petite fille qui ne comprend rien à ce que lui demande le juge, lequel cherche pourtant à se mettre à sa place en recourant à la science ethnographique, en toute sincérités.

9 D'une manière générale, il y a ceux qui sont du côté du sensible comme les artistes et qui subissent la loi du langage articulé. Ce ne sont donc pas nécessairement des victimes mais des résistants face à l'exigence langagière de l'articulation en signes qui trouve son sommet dans la norme de délibération.

Dès lors pour Lyotard, s'il y a une politique du différend, elle place en première ligne les artistes et les penseurs. Pourquoi eux et non pas ceux qui militent pour une démocratie toujours élargie, comme le prône Rancière? C'est que les sans-part, ces nouveaux prolétaires, décrits par Rancière dans $L a$ Mésentente parce qu'ils inaugurent une nouvelle place publique et donc un autre partage du sensible, inventent de nouveaux mobiles à l'action, de nouvelles revendications et de nouvelles argumentations. Mais parce qu'ils ont à convaincre le public de la justesse de leur mouvement, ils entrent nécessairement sous l'orbe de la norme délibérative. D'ailleurs, en cas de succès, ils rejoindront le monde légitime de ceux qui prennent part à la vie politique, sans reste. Les anciennes discriminations vont sauter, les femmes seront citoyennes à part entière, etc. Mais les sans-part auront dû se soumettre au principe de raison, ce n'est qu'à ce prix que leur révolte acquerra une légitimité. Je considère donc que Rancière a enrichi le chapitre de Lyotard consacré au délibératif, La Mésentente devenant une partie du Différend mais avec les limites de la norme délibérative. On pourrait dire, en reprenant le constat du Marx de La Question juive : il condamne les sauvages, les païens et autres monothéistes à faire fi de leur communauté et à devenir des individus monadiques : démocratiques ${ }^{6}$.

11 Qu'attendre alors des penseurs et des artistes ? Il faut déjà avoir conscience qu'il n'y a pas de science de la communication interculturelle. C'est le paradoxe russellien de l'ensemble de tous les ensembles: l'ensemble de tous les ensembles n'est pas contenu 
dans son ensemble. Il n'y a donc pas de point de surplomb possible. C'est le grand apport de Cl. Lefort, ancien élève de Merleau-Ponty, à Lyotard, qui permettait à Lefort dans les années 1960-1970, contre les fantasmes d'emprise absolue de Castoriadis, de critiquer l'idée d'un parti révolutionnaire tout sachant, au-dessus de la lutte des classes. Un parti d'ingénieurs sociaux néo-bolcheviques.

Pourtant on rétorquera que Lyotard a bien décrit trois pôles de légitimité, comme s'il les surplombait. Mais ce faisant il n'a pas la prétention d'inclure son discours dans ce qu'il décrit. Ce fut au contraire l'illusion de la philosophie hégélienne de l'esprit: l'esprit s'engendrant de ce qui n'est pas lui, le sensible, pour, à partir de cet intérieur, passer à l'extérieur en intégrant toutes ses figures historiques et philosophiques dépassées vers une totalité de l'identité à soi-même. On peut considérer Le Différend comme une systématique machine de guerre contre l'hégélianisme. Pourtant la négativité hante l'œuvre de Lyotard, c'est la mort anamorphosée qui se trouve au cœur du tableau d'Holbein: Les Ambassadeurs, tableau qui sert de frontispice à la thèse de Lyotard: Discours, Figure. Si la mort comme négativité est au cœur de l'ouvrage, c'est que le discours pour désigner un référent à l'extérieur de lui-même doit en quelque sorte s'en écarter, le mettre à mort comme objet qu'il désigne. La mort est donc au cœur du discours empirique et cognitif, donc au cœur de la science comme objectivation délibérante, comme elle est présente au cœur du système linguistique des signes du fait de leur articulation horizontale (Saussure), et finalement comme elle l'est encore au cœur de l'inconscient, en particulier dans la dénégation freudienne, quand dire non, c'est dire oui. Lyotard est donc tout contre Hegel.

Ces trois modes de la négativité rendent possible en apparence une totalisation sous la forme d'une encyclopédie de l'esprit des cultures. Et pourtant il n'y a pas pour Lyotard d'histoire de la raison, car il n'y a pas de raison dans l'histoire. La preuve : les génocides $\mathrm{du} \mathrm{xx}^{\mathrm{e}}$ siècle et les millions d'existences disparues. Ces existences qui, accumulées, constituent le trou noir de la raison parce que le règne de la phrase constative s'arrête devant elles.

Cette butée nous oblige à considérer toutes les discontinuités de l'histoire passée : on ne donnera jamais raison du fait que, comme le montre P. Veyne dans son livre sur l'adoption par Constantin du christianisme ${ }^{7}$, qu'un empereur romain, pour des motifs définitivement inaccessibles et irrationnels, de l'ordre de la grâce, ait pu abandonner le paganisme.

15 Second niveau de différends : entre les genres de discours eux-mêmes. Ces genres de discours dont la différenciation n'a pas cessé depuis la norme narrative. On peut faire une comparaison, fragile comme toute comparaison. Si la norme narrative a pu synthétiser tous les genres de discours possibles: le constatif (au sens général de la connaissance sur les hommes, la nature, la société), l'éthique (comment se conduire ?), l'esthétique (comment apparaître ?), le théologique, etc., il y a depuis une sorte de Big Bang ouvrant une différenciation entre les genres de discours qui n'a pas cessé et qui a été accélérée quand la norme délibérative s'est imposée, déjà avec la démocratie athénienne et son utilisation de l'écriture alphabétique (la géométrie, l'histoire, la géographie, la rhétorique, la logique, la philosophie, etc.).

De ce point de vue, le kantisme est une étape essentielle de la reconnaissance d'une raison en archipel : la légitimité intrinsèque de chaque Critique institue à chaque fois comme une île. Entre ces îles, des ponts pourront être édifiés, mais dans un second temps. Chaque Critique édifie un appareillage transcendantal spécifique : le commun 
entre elles, c'est donc cet appareillage transcendantal, l'architectonique de la raison. Mais on ne saurait déduire de la nécessité de l'une, la nécessité de l'autre, par exemple : l'esthétique (Critique de la faculté de juger) de la connaissance a priori (Critique de la raison pure). Résultat : quand le sujet de l'esthétique déclare d'une chose qu' « elle est belle ", il ne le sait, à proprement dit, pas. Un sentiment de plaisir à l'occasion d'une chose n'est pas une connaissance attribuable à un objet. La beauté n'est pas un savoir : il n'y aura pas de science du beau.

17 C'est la raison pour laquelle quand Lyotard exposera quelques principes de la raison «post-moderne» et proposera au Centre de Création industrielle du Centre G. Pompidou, en 1985, le programme d'une exposition sur le numérique et le virtuel, Les Immatériaux, la mise en espace respectera encore plus la disposition en archipel. Et développera un appareillage transcendantal spécifique à partir de la racine sanscrite mat: matière, matériaux, matériel, matrice, maternité, etc. C'est probablement la première tentative philosophique pour prendre en considération la surface d'inscription comme dimension incontournable de l'appareillage transcendantal, après L'origine de la géométrie de Husserl.

Pour décrire ce mouvement de différenciation, Lefort parlera de désintrication : pour lui, en rupture avec le théologico-politique, la démocratie va de pair avec la désintrication de la loi, du savoir et du pouvoir. Ce qui entraîne une différenciation des secteurs de la réalité, des savoirs nécessaires, des registres du pouvoir et de la loi. Chaque secteur se voit analysé selon sa logique, au risque d'un recouvrement et d'une omnipotence. Que l'on constate avec Foucault où tout relève du pouvoir, avec les néolibéreaux du marché, avec Al Quaïda, du théologique islamique, comme auparavant, avec les marxistes, où tout était politique ou aujourd'hui, avec Stiegler, où tout est technique, etc. La démocratie serait ce régime, à l'épreuve de l'indétermination des repères symboliques, où la différenciation des genres de discours est déclarée légitime, où les philosophes ont constamment à remettre dans le rang tel ou tel régime de discours, qui, sinon se ferait passer pour l'unique, dans une totalité dont il détiendrait les clefs. Ce passage hors limite d'un genre de discours (et du secteur de réalité qui est le sien) peut être qualifié de métaphysique et il est la cause de très nombreux différends. Par exemple : qui est légitimé à parler d'art ? Peut-on caricaturer le Dieu d'autrui?

19 Troisième dimension du différend: entre les phrases elles-mêmes. Récapitulons : les normes de légitimité imposent leur ordre aux genres de discours, ces derniers attirent les phrases dans leur orbite. Par exemple : le genre de discours esthétique imposera aux phrases un enchaînement spécifique. Comment concevoir l'articulation entre les trois ordres du différend? Si l'on reconstruit l'appareil transcendantal lyotardien à partir de la phrase, alors on a la surprise de constater qu'il s'agit de l'élément le moins langagier, le plus « sensible », malgré les apparences. C'est que, même si une phrase est un univers qui positionne en les articulant un référent, une signification, un destinataire et un destinateur, c'est comme le ferait un phénomène météorologique un peu exceptionnel quand il nous arrive dessus, nous qui devenons ses destinataires. Comment allons-nous enchaîner? Par une phrase cognitive? "C'est un nimbo-cumulus ", par une phrase esthétique: "c'est sublime!», par une phrase d'ordre: "protégez-vous, l'orage arrive!». C'est dire qu'une phrase, c'est ce qui arrive et qui engage la question de ce quod? qu'elle est. On appellera donc phrase l'événement ou le réel qui interroge. On peut rapprocher la phrase lyotardienne des data sensibles kantiens, que les formes a 
priori de la sensibilité (espace, temps) synthétisent. Mais ces data ne sont pour Kant que de la matière au sens de l'hylémorphisme aristotélicien, quelque chose comme un tas de sable sans forme. On ne voit pas un tas de sable s'avancer comme question. Il doit y avoir dans la phrase lyotardienne une sorte d'aventure, de surprise ou de résistance par rapport à la réalité établie (ce qu'établissent comme réalités les genres de discours). Ce serait la distinction établie par Rosset entre le réel et la réalité. D'où le privilège, chez Lyotard, de l'art et de la peinture en particulier : comment enchaîner sur telle œuvre?

Le problème que rencontre cette ontologie du singulier où l'être s'appelle "phrase ", engageant en outre à chaque fois une certaine spatialité et une temporalité internes, c'est celui du temps et de la liberté. Dans un atomisme simple (Épicure, Lucrèce), il y va du clinamem (déviation légère, déclinaison) pour que les atomes ne tombent pas tout à fait en chute libre, mais fassent un petit écart qui est essentiel. Pour Lyotard, d'une part, l'être est temps, mais l'être, toujours singulier, du fait de son incomplétude (le réel n'est pas la réalité) exige que l'univers qu'il comporte soit explicité, par un autre être, une autre phrase, qui enchaîne en le portant à l'explicitation. Mais elle-même, cette seconde phrase, est césurée, elle entraînera donc la nécessité d'une troisième phrase. Dès lors, cet enchaînement toujours relancé est nécessaire et improbable : il y a un différend irréductible entre la seconde phrase et la première sur laquelle elle enchaîne, même si les genres de discours qui s'imposent aux phrases réduisent la part d'improbabilité. J'ai proposé dans L'époque des appareils d'analyser l'appareil cinématographique et les enchaînements entre phrases-images d'un film, à partir de l'axiomatisation élaborée par Le Différend. Un "bon" film serait un film où chaque phrase-image est un quod? comme dans le dernier Monteiro.

21 La philosophie de la phrase fait de chaque locuteur un récepteur (au sens d'une esthétique de la réception) ou un destinataire. Comme si la souveraineté initiale de la narration (le récit) était conservée (comme chez Benjamin d'ailleurs): ce sont les phrases qui «nous » font et non l'inverse. Ce qui a une conséquence importante : il n'y aura pas de dernière phrase. Donc il n'y aura pas de tribunal de l'histoire. Ce qui implique à mon sens que l'hypothèse d'une "vie nue " au sens d'Agamben doit être reconsidérée : l'homo sacer romain n'était-il pas phrasé à l'intérieur d'un rituel ?

Dans le même ordre d'idée, l'hypothèse lyotardienne d'une force des faibles, dont on peut donner une lecture sociologique, récuserait le thème d'un "paria absolu », d'une singularité absolument hors du langage, donc absolument en dehors des rapports sociaux. C'est l'expérience du concentrationnaire qui, si les bourreaux lui en laissent le temps, découvre la double organisation du camp comme condition de la survie. Dans la descente aux enfers constituée par la série : camp de transit pour immigrés libres (cf. Sangatte), camp de réfugiés, camp d'internement, camp de concentration, camp d'extermination, je ne suis pas sûr que la lecture doive se faire à partir de ce dernier. Le critère, c'est la possibilité d'une révolte. Laquelle suppose un minimum de reconnaissance entre les singularités. Le paradoxe est le suivant: la lutte pour la reconnaissance suppose toujours un minimum de reconnaissance ${ }^{8}$.

Il y a une limite qui pose néanmoins problème : il peut y avoir génocide sans différend. Non pas en ce qui concerne l'extermination des juifs d'Europe ${ }^{9}$. Mais considérons le génocide rwandais : des paysans hutus assassinent en masse des paysans tutsis. Même normes de légitimité (la narration, la révélation), même cosmétique (même surface d'inscription: le Livre), même religion, j'ajouterai, ce qui n'est pas un critère 
lyotardien : même langue, même probable origine. Le cas cambodgien serait un peu différent : visiblement les choses se sont articulées à partir de l'opposition campagne/ ville et les lettrés (au sens large) ont été largement persécutés. On peut faire l'hypothèse que les Khmers rouges aient voulu forger une sorte de communauté paysanne purifiée de ses éléments dégénérés (sachant lire) et en éliminant les nonkhmers.

Par ailleurs, d'autres distinctions seraient nécessaires pour rendre compte des guerres de religion, entre religions partageant la même norme (révélation), la même surface d'inscription (le Livre), le même rapport au corps (l'incarnation). C'est évidemment le cas entre catholiques et protestants et plus largement entre eux et les orthodoxes. Ici, il faut introduire la question de l'image (refus des images par la Réforme / icônes byzantines / imagerie du catholicisme).

Il faut introduire alors le concept de différend cosmétique.

\section{BIBLIOGRAPHIE}

Agier Michel, « Le Gouvernement humanitaire et la politique des réfugiés », in Laurence Cornu et Patrice Vermeren (dir.), La philosophie déplacée. Autour de J. Rancière, colloque de Cerisy, Paris, Horlieu éditions, 2006.

Benjamin Walter, «Thèses sur le Concept de philosophie de l'histoire », in Euvres complètes, t. 3, Paris, Gallimard, 2000.

Déotte Jean-Louis, Qu'est-ce qu'un Appareil ? Benjamin, Lyotard, Rancière, Paris, L'Harmattan, 2006.

Kant Emmanuel, Critique de la Faculté de juger, A. Philonenko (trad.), Paris, Vrin, 1965.

Lefeuvre-Déotte Martine, L'Excision en procès. Un Différend culturel ?, Paris, L'Harmattan, 1997.

Lyotard Jean-François, Le Différend, Paris, Minuit, 1983.

Lyotard Jean-François, Heidegger et les «juifs », Paris, Galilée, 1988.

Lyotard Jean-François, La Guerre des Algériens, Paris, Galilée, 1989.

Veyne Paul, Quand notre Monde est devenu chrétien (312-394), Paris, Albin Michel, 2007.

\section{NOTES}

1. Jean-François Lyotard, Le Différend, Paris, Minuit, 1983.

2. Walter Benjamin, «Thèses sur le Concept de philosophie de l'histoire », in Euvres complètes, t. 3, Paris, Gallimard, 2000.

3. Emmanuel Kant, Critique de la Faculté de juger, A. Philonenko (trad.), Paris, Vrin, p. 127.

4. Jean-François Lyotard, La Guerre des Algériens, Paris, Galilée, 1989.

5. Martine Lefeuvre-Déotte, L'Excision en procès. Un Différend culturel ?, Paris, L'Harmattan, 1997.

6. Jean-Louis Déotte, Qu'est-ce qu'un Appareil ? Benjamin, Lyotard, Rancière, Paris, L'Harmattan, 2006.

7. Paul Veyne, Quand notre Monde est devenu chrétien (312-394), Paris, Albin Michel, 2007. 
8. Michel Agier, "Le Gouvernement humanitaire et la politique des réfugiés ", in Laurence Cornu et Patrice Vermeren (dir.), La philosophie déplacée. Autour de J. Rancière, colloque de Cerisy, Paris, Horlieu éditions, 2006.

9. Jean-François Lyotard, Heidegger et les « juifs », Paris, Galilée, 1988.

\section{AUTEUR \\ JEAN-LOUIS DÉOTTE}

Philosophe français, professeur à l'université Paris 8 